\title{
The blow flies recorded from Pakistan, with the description of one new species (Diptera: Calliphoridae)
}

\author{
Hiromu KURAHASHI ${ }^{1)}$ and Mohammad AFZAL ${ }^{2)}$ \\ ${ }^{1)}$ Taxonomy and Ecology Laboratory, Department of Medical Entomology, National Institute of Infectious \\ Diseases, Toyama 1-23-1, Shinjuku-ku, Tokyo, 162-8640 Japan \\ ${ }^{2)}$ Pakistan Museum of Natural History, Islamabad, Pakistan
}

(Received: 20 February 2002; Accepted: 5 March 2002)

Key words: blow fly, Calliphoridae, fauna, new species, Onesia kiyoshii sp. nov., Pakistan

\begin{abstract}
Fourty eight calliphorid species belonging to eighteen genera are recorded from Pakistan including the description of a new species, Onesia kiyoshii sp. nov. Thirty-seven species are new to Pakistan.
\end{abstract}

\section{INTRODUCTION}

The adults of Calliphorid blow flies are often known to be of great medical and veterinary importance due to their roles in myiasis and disseminating agents of infectious diseases. However, very little information is available for the family Calliphoridae from Pakistan except for records of several species in the previous works (Senior-White et al., 1940; James, 1977).

During the field survey of the "Zoogeographical Studies on the Medically Important Diptera in Southwest Asia" in 1986-89, we made an excellent collection of the blow flies although this would be covered only a part of whole fauna, indeed. We examined the collected materials and recorded 48 species belonging to 18 genera of Calliphoridae. Three of Onesia and one of Polleniopsis were considered to be new to science. One of these new species is described here in this paper. But, the other three will be described soon in a separate paper after detailed examinations. We tried to discuss the blow fly fauna of Pakistan. Most fly species seem to be so-called Oriental elements (54\%), and then Palaearctic (27\%) and Afrotropical ones (4\%). Seven species (15\%) including four new species seem to be endemic in Pakistan and its adjacent areas. Thirty-seven species are new to Pakistan and their names and country name in the distribution section are preceded by asterisk except for the new species.

Abbreviations for institutions housing specimens are as follows: MNHN, Musee Nationale d'Histoire Naturelle, Paris; NIID, Reference Museum, Department of Medical Entomology, National Institute of Infectious Diseases, Tokyo; NSMT, National Science Museum (Natural History), Tokyo; OUAV, Obihiro University of Agriculture and Veterinary 
Medicine, Obihiro.

Locality names of specimens examined are cited as shown on the label of specimens. Countries given under distribution section are listed roughly in east-west sequence. Terminology mainly follows Senior-White et al. (1940) and measurement of frons was made in a similar manner to Fan (1965). Frons index is the same as Fan's index of frons.

\section{Subfamily Ameniinae \\ Tribe Catapicephalini}

${ }^{\star}$ Catapicephala pattoni Senior-White, Aubertin et Smart, 1940

Specimens examined. $18 \AA$ 19으, Buttgram, 1,100 m, 24.vii.1988, H. Kurahashi (NIID); 1ㅇ, Murree, 2,000 m, 26.vi.1989, H. Kurahashi (NIID); 1ठึ, Tret, $22 \mathrm{~km} \mathrm{~S} \mathrm{of} \mathrm{Murree,} 500 \mathrm{~m}$, 26.vi.1989, H. Kurahashi (NIID); 2ð 1q, Murree, 2,000 m, 21.vii.1988, H. Kurahashi (NIID); $10 \delta^{\star}$ 14오. Swat, Marghazar, 1,400 m, 8.viii.1987, H. Kurahashi (NIID); $1 \delta^{\star}$, Miandam, 1,300 m, 4.viii.1988, H. Kurahashi (NIID); 5ð, Nathiagali, 2,200 m, 25-30. vii.1988, H. Kurahashi (NIID); 2ð, Shangulia Pass, 1,700 m, 9.viii.1988, H. Kurahashi (NIID).

Distribution. India and *Pakistan.

\section{Subfamily Calliphorinae \\ Tribe Calliphorini}

\section{${ }^{*}$ Calliphora chinghaiensis Van et Ma, 1978}

Specimens examined. 1ठ, Khunjeranb Pass, 4,700 m, 2.viii.1987, H. Kurahashi (NIID); 1ठ, Khunjerab Pass, 4,940 m, 20.viii.1988, T. Hayashi (NIID).

Distribution. China (Chinghai, Tibet, Yunnan), Nepal and *Pakistan.

\section{${ }^{*}$ Calliphora himalayana Kurahashi, 1994}

Specimens examined. 1ठ, Khunjerab Pass, 4,700 m, 2.viii.1987, K. Kanmiya (NIID); 1 , Babusar, 30 km S Chilas, 3,000 m, 5.viii.1987, S. Shinonaga (NIID).

Distribution. Nepal and *Pakistan.

*Calliphora uralensis Villeneuve, 1922

Specimens examined. $3 \delta^{\Uparrow} 209$, Khunjerab Pass, 4,940 m, 20.viii.1988, K. Kamimura

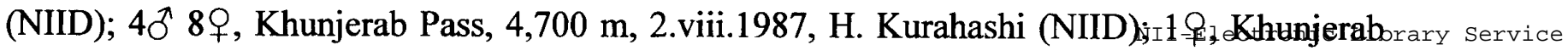


Pass, 4,500 m, 1,3.vii.1989, H. Kurahashi (NIID); 5ठ 1우 Khunjerab Pass, 4,940 m, 18, 20.viii.1988, T. Hayashi (NIID); $1{ }^{\curvearrowright}$, Chilas, 700 m, 4.viii.1987, S. Shinonaga (NIID).

Distribution. China, Mongolia, *Pakistan, Russia and Europe.

Calliphora vicina Robineau-Desvoidy, 1830

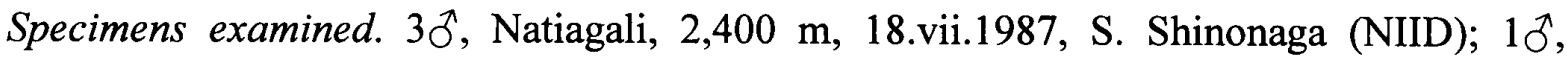
Murtazabad, $20 \mathrm{~km} \mathrm{~S}$ of Hunza, 1,800 m, 30.vii.1987, H. Kurahashi (NIID); 1 $\delta$, Chilas Babusar, $30 \mathrm{~km}$ S of Chilas, 3,000 m, 5.viii.1987, H. Kurahashi (NIID); $1 \delta$, Chitral, 1,506 m, 3.x.1986, H. Kurahashi (NIID); 2ð̄, Gilgit, 1,500 m, 26.vii.1987, S. Shinonaga \& H. Kurahashi (NIID); 1 9 , Saif-ul-Malook Lake, 2,700 m, 20.vii.1987, H. Kurahashi (NIID); $1 \delta$ 1 , N Naltar, $20 \mathrm{~km}$ N Gilgit, 3,000 m, 28.vii.1987, S. Shinonaga \& T. Inaoka (NIID); $1 \delta 2$, Kalash valley, 1,800 m, 4.x.1986, H. Kurahashi (NIID); $2 \delta 3 \%$, Swat, Ushu, $10 \mathrm{~km} \mathrm{~N} \mathrm{of}$ Kalam, 2,000-2,800 m, 10.viii.1987, S. Shinonaga \& H. Kurahashi (NIID); 1ð̊, Naran, Kagan Valley, 2,000 m, 22.vii.1987, S. Shinonaga (NIID); 2ㅇ, Hunza, Karimabad, 2,400 m, 31.vii.-1.viii.1987, H. Kurahashi (NIID); 1ठ, Hunza, Ultar gracier, 2,500 m, 31.vii.1987, S. Shinonaga (NIID); $12 \delta^{\Uparrow} 1$, Gulmit, $35 \mathrm{~km}$ N of Gilgit, 1.viii.1987, S. Shinonaga \& H. Kurahashi (NIID); 2ð 2q, Ziarat, 17,18.ix.1988, M. Iwasa (NIID); $1 \delta$ 1 + , Nathiagali, 2,400 m, 17-18.vii.1987, H. Kurahashi (NIID); 4ð 1q, Nathiagali, 2,200 m, 25-30.vii.1988, H.

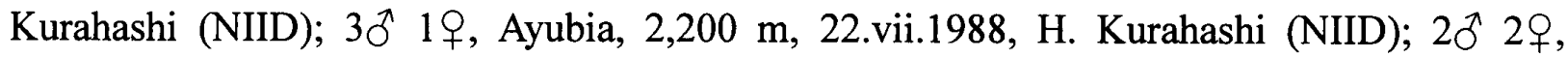
Thandani, 2,400 m, 1.viii.1988, H. Kurahashi (NIID); 29, Gilgit to Gakuch, Gurapur, 1,600 m, 5,7.vii.1989, H. Kurahashi (NIID); 19, Khunjerab Pass, 4,700 m, 2.viii.1987, H. Kurahashi (NIID); 4으. Khunjerab Pass, 4,500 m, 1,3.vii.1989, H. Kurahashi (NIID); 2 , Nathiagali, 2,500 m, 26-28.vi.1989, H. Kurahashi (NIID); 10 1온. Nathiagali, 2,000 m, 10-11.vii.1989, H. Kurahashi (NIID); $9 \delta$ 59, Pasu, 2,700 m, 2-3.vii.1989, H. Kurahashi (NIID); $1 \hat{\jmath} 1$ 1 , Khunjerab Pass, 4,940 m, 20.viii.1988, T. Hayashi \& K. Kamimura (NIID); $1 \delta^{\Uparrow} 2$, Kashmir, Ayubia Nat. Park, 2,200 m, 25-30.vii.1988, S. Shinonaga (NIID).

Distribution. Mongolia, China, Australia, New Zealand, Nepal, India, Pakistan, Saudi Arabia, Russia, Europe, Mauritius, South Africa, Canary Is., North America, Uruguay, Argentina, Chile (South to Tierra del Fuego) and Hawaiian Is.

\section{*Calliphora vomitoria (Linnaeus, 1758)}

Specimens examined. $6 \delta^{\lambda} 8$, Natiagali, $10 \mathrm{~km} \mathrm{~N}$ Murree, 2,400 m, 18.vii.1987, S. Shinonaga (NIID); 1q, Ayubia, Dunga Gali, 17.vii.1988, K. Kanmiya (NIID); 1q, Kashmir, Ayubia Nat. Park, 2,200 m, 25-30.vii.1988, S. Shinonaga (NIID); $0 ð 2$, Kagan Valley, Naran, 2,000 m, 22.vii.1987, S. Shinonaga (NIID); 3q, Kalash valley, 1,800 m, 4.x.1986, H. Kurahashi (NIID); 19, Swat, Ushu, $10 \mathrm{~km} \mathrm{~N}$ of Kalam, 2,000 m, 10.viii.1987, H. Kurahashi (NIID); $1 \hat{\jmath}$, Gilgit, Naltar, $20 \mathrm{~km}$ N of Gilgit, 3,000 m, 28.vii.1987, H. Kurahashi (NIID); $1 \hat{\mathrm{N}} \hat{\mathrm{N}}$ 
1․ Thandani, 2,400 m, 1.viii.1988, H. Kurahashi (NIID); 30 5오, Nathiagali, 2,500 m, 26-28.vi.1989, 10-11.vii.1989, H. Kurahashi (NIID); 7ơ 59, Nathiagali, 2,400 m, 17-18. vii.1987, H. Kurahashi (NIID); 4 $\delta^{\Uparrow}$, Nathiagali, 2,300 m, 25-30.vii.1988, H. Kurahashi (NIID); 3ㅇ, Saif-ul-Malook Lake, 2,700 m, 20.vii.1987, H. Kurahashi (NIID); 1ठ 3ㅇ, Lalazar, 16 km W Naran, 2,700 m, 21.vii.1987, S. Shinonaga (NIID).

Distribution. Japan, Korea, Taiwan, Philippines (Luzon, Mindanao), Mongolia, China, Thailand, Nepal, India, *Pakistan, Afghanistan, Europe, Morocco, Canary Is., N. America and Hawaiian Is.

${ }^{*}$ Cynomya mortuorum (Linnaeus, 1761)

Specimens examined. 2ð̄, Khunjerab Pass, 4,940 m, 20.viii.1988, K. Kamimura (NIID). Distribution. China, Mongolia, *Pakistan, Russia and Europe.

*Polleniopsis sp.

This form is most probably a new species and will be described in a separate paper.

Specimens examined. $1 \delta$, Ushu, $10 \mathrm{~km} \mathrm{~N}$. Kalam, 2,800 m, 10.viii.1987, S. Shinonaga (NIID); 1ठ, Tandani, 26 km N. Abbottabad, 2,400 m, 1.viii.1988, S. Shinonaga (NIID).

Distribution. *Pakistan.

\section{*Onesia sp. 1}

This form is most probably a new species and will be described in a separate paper. Specimens examined. 4ð, NWF Prov., Shangula Pass, 1,700 m, 9.viii.1988, H. Kurahashi (NIID).

Distribution. *Pakistan.

*Onesia sp. 2

This form is most probably a new species and will be described in a separate paper. Specimens examined. 10ึ, NWF Prov., Nathiagali, $10 \mathrm{~km}$ N Murree, 2,400 m, 18.vii.1987, S. Shinonaga (NIID); 1ð̊, Naran, Kagan V., 1,800 m, 22.vii.1987, K. Kanmiya (NIID). Distribution. *Pakistan.

Specimens examined. $3 \delta 2 ᄋ$, Kalam, $1,800 \mathrm{~m}$, 5.viii.1988, H. Kurahashi (NIID); $1 \delta^{\Uparrow}$,

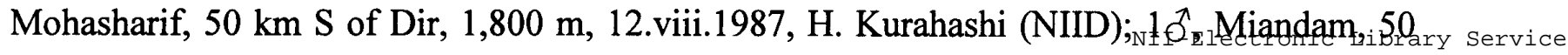


Vol.53 Suppl.2, 2002

km N Mingora, 1,700 m, 4.viii.1988, S. Shinonaga (NIID); 1ठ, Darora, 20 km S Dir, 12.viii. 1987, K. Kanmiya (NIID); 3ơ 11 , Mingora, 1,000 m, 8-10.viii.1988, H. Kurahashi (NIID);

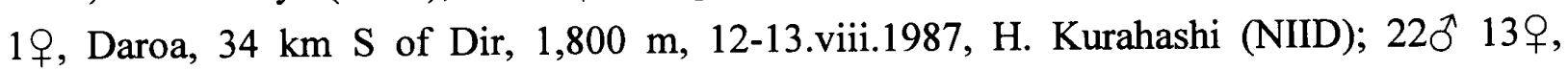
Miandam, 1,300 m, 4.viii.1988, H. Kurahashi (NIID); 4, Shangula Pass, 1,700 m, 9.viii. 1988, H. Kurahashi (NIID).

Distribution. Japan, China and *Pakistan.

*Onesiomima pamirica Rohdendorf, 1962

Specimens examined. 5ठ 6우, Khunjerab Pass, 4,500-4,700 m, 2.viii.1987, 1, 3.vii.1989, H. Kurahashi (NIID).

Distribution. China, *Pakistan, Tadzhikistan and Kirgizstan.

*Melinda sugiyamai Kurahashi et Thapa, 1994

Specimens examined. 19, Daroa, 34 km S of Dir, 1,800 m, 12-13.viii.1987, H. Kurahashi (NIID); 1ठ, Darora, 20 km S Dir, 12.viii.1987, K. Kanmiya (NIID).

Distribution. Nepal and *Pakistan.

*Melinda scutellata (Senior-White, 1923)

Specimens examined. $1 \delta$ 19, Kashmir, Aybia Nat. Park, 2,200 m, 25-30.vii.1988, S. Shinonaga (NIID); 1 , Daroa, $34 \mathrm{~km} \mathrm{~S}$ of Dir, 1,800 m, 12-13.viii.1987, H. Kurahashi

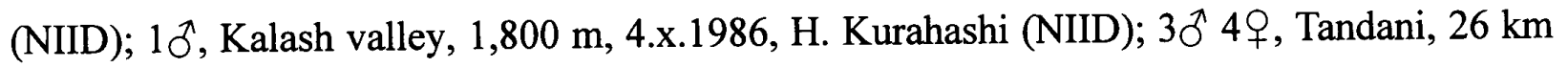
N. Abbottabad, 2,400 m, 1.viii.1988, S. Shinonaga \& H. Kurahashi (NIID); $1 \delta^{\Uparrow}$, Nathiagali, $10 \mathrm{~km}$ N of Murree, 2,400 m, 17-18.vii.1987, H. Kurahashi (NIID); $1 \delta^{\wedge} 1$, Nathiagali, 2,200 m, 25-30.vii.1988, H. Kurahashi (NIID); 1ðð, Miandum, 1,800 m, 7.viii.1987, K. Kanmiya (NIID); $1 \delta^{\pi}$, Ayubia, 2,200 m, 22.vii..1988, H. Kurahashi (NIID).

Distribution. Malaysia, Myanmar, Nepal and *Pakistan.

\section{Tribe Luciliini}

*Hemipyrellia ligurriens (Wiedemann, 1830)

Specimens examined. $1 \delta$ ๙ 1 , Abbottabad, 1,100 m, 2.viii.1988, H. Kurahashi (NIID); $1 \hat{\delta}$, Mansehra, 1,000 m, 2.viii.1988, S. Shinonaga (NIID); 2ð̃, Buttgram, 1,100 m, 24.vii.1988, H. Kurahashi (NIID).

Distribution. Japan, Taiwan, Philippines, China, Thailand, Malaysia, Indonesia, Nepal, India and *Pakistan. 
*Hemipyrellia pulchra (Wiedemann, 1830)

Specimens examined. $4{ }^{\wedge} 19$, Thatta, Haleji Lake, 10 m, 21,23,29.vii..1990, 6,11,12.viii. 1990, K. Kamimura (NIID).

Distribution. Thailand, India and *Pakistan.

${ }^{*}$ Lucilia cuprina (Wiedemann, 1830)

Specimens examined. 1 , , Murree, 1,300 m, 21.vii.1988, S. Shinonaga (NIID); 101 , Kohat, 8.ix.1988, M. Iwasa (NIID); 19, Tandani, $20 \mathrm{~km} \mathrm{~N}$ of Abbottabad, 2,400 m, 1.viii. 1988, S. Shinonaga (NIID); + , Kalam, 1,800 m, 5.viii.1988, H. Kurahashi (NIID); 1 , Hyderabad, 6.x.1986, T. Hayashi (NIID); 10ð̊ 2q, Dasu to Besham, Keyal, 600 m, 9.vii.1989, H. Kurahashi (NIID); 4ð๋, Mansehra, 19.vii.1987, H. Kurahashi (NIID); 4ðં, Ayubia, 2,200 m, 22.vii.1988, H. Kurahashi (NIID).

Distribution. Taiwan, Philippines (Luzon, Palawan, Mindanao), China, Vietnam, Laos, Cambodia, India, *Pakistan and N. Africa. Partly invades southern parts of Holarctic Region such as Korea, Japan, Spain, North Carolina and California.

\section{*Lucilia papuensis Macquart, 1842}

Specimen examined. 1 , Nathiagali, $10 \mathrm{~km} \mathrm{~N}$ of Murree, 2,400 m, 17-18.vii.1987, H. Kurahashi (NIID).

Distribution. Japan, Taiwan, Philippines (Luzon, Palawan, Clion, Leyte, Mindanao), China, Thailand, Malaysia (Malaya, Borneo), Indonesia (Maluku), Papua New Guinea, Australia (Queensland, New South Wales), India, Sri Lanka, *Pakistan and Micronesia.

\section{${ }^{*}$ Lucilia porphyrina (Walker, 1856)}

Specimens examined. $32 \delta^{\Uparrow} 14$, Nathiagali, $10 \mathrm{~km} \mathrm{~N}$ of Murree, 2,400 m, 17-18.vii.1987, S. Shinonaga, K. Saito \& H. Kurahashi (NIID); 19, Swat, Miandam, 1800 m, 7.viii.1987, H. Kurahashi (NIID); 1 , Kagan Valley, Balakot, 1,270 m, 23.vii.1987, H. Kurahashi (NIID); 1q, Daroa, $34 \mathrm{~km}$ S of Dir, 1,800 m, 12-13.viii.1987, H. Kurahashi (NIID); 2q, Kalash Valley, 1800 m, 4.x.1986, H. Kurahashi (NIID); 2ð’, Kagan Valley, Naran, 2,000 m, 22.vii. 1987, S. Shinonaga (NIID); 2ð, Miandam, 4.viii.1988, H. Kurahashi (NIID); $1 \delta$, Buttgram, 1,100 m, 24.vii.1988, H. Kurahashi (NIID); 2ð 4우, Ayubia, 2,200 m, 22.vii.1988, 25-30.vii. 1988, S. Shinonaga \& H. Kurahashi (NIID); 1ठ, Kagan Valley, Awa, 22.vii.1987, H. Kurahashi (NIID); $3 \delta 2$, Murree, 1,300-2,000 m, 21.vii.1988, S. Shinonaga \& H. Kurahashi

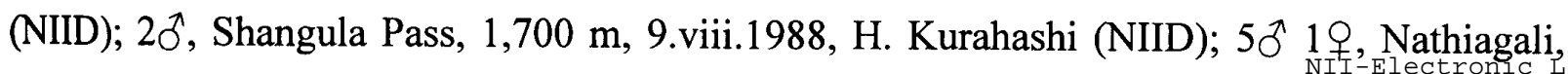


2,500 m, 26-28.vi.1989, H. Kurahashi (NIID); $1 \delta 1$, Dasu to Besham, Keyal, 600 m, 9.vii. 1989, H. Kurahashi (NIID).

Distribution. Japan, Korea, China, Taiwan, Philippines (Palawan), Vietnam, Thailand, Malaysia (Malaya, Borneo), Indonesia (Sumatra, Java), Papua New Guinea (New Guinea, Manus I., Mussau I., New Britain, New Irleand), Australia (Queensland), Bangladesh, Nepal, India, Sri Lanka and *Pakistan. Widely distributed in the Indo-Australian and East Palaearctic regions.

\section{Lucilia sericata (Meigen, 1826)}

Specimens examined. $4 \delta 1$, Nathiagali, $10 \mathrm{~km} \mathrm{~N}$ of Murree, 2,400 m, 18.vii.1987, S. Shinonaga (NIID); 1 $\delta$, Shangula Pass, 1,700 m, 9.viii.1988, H. Kurahashi (NIID); 33 $\delta 18$ ․ Chitral, 1,506 m, 3.x.1986, H. Kurahashi (NIID); 2ð 2q, Ziarat, 16-17.ix.1988, M. Iwasa (NIID); $1 \delta 3$, Kalash Valley, 1,800 m, 4.x.1986, H. Kurahashi (NIID); 5ð 2q, Gulmit, 35 $\mathrm{km} \mathrm{N}$ of Gilgit, 1.viii.1987, S. Shinonaga \& T. Inaoka (NIID); 19, 8 km E of Gilgit, 26.vii. 1987, K. Kanmiya (NIID); 2ð 2ㅇ, Fort Munro, 13.ix.1988, 15.ix.1988, M. Iwasa (NIID); 5ð̋, Dih, 18-19.viii.1988, K. Kamimura \& T. Hayashi (NIID); $1 \delta^{\star}$, Ushu, $10 \mathrm{~km} \mathrm{~N}$ of Kalam, 2,700 m, 6.viii.1988, S. Shinonaga (NIID); 1ð, Kalam, 2,400 m, 5.viii.1988, S. Shinonaga (NIID); $2 \widehat{\jmath}$, Naltar, $20 \mathrm{~km} \mathrm{~N}$ of Gilgit, 3,000 m, 28.vii.1987, S. Shinonaga \& T. Inaoka (NIID); $2 \delta^{\star}, 8 \mathrm{~km}$ E of Gilgit, 26.vii.1987, K. Kanmiya (NIID); $1 \delta^{\Uparrow} 3$, Hunza, Ultar Gracier, 2,500 m, 31.vii.1987, S. Shinonaga (NIID); 2ð’, Swat Valley, Kalam, 1,800 m, 9.viii.1987, H. Kurahashi (NIID); 1ㅇ, Kagan Valley, Awa, 22.vii.1987, H. Kurahashi (NIID); $1 \delta^{\star}$, Kawai-Dasu, 1,200 m, 22.vii.1987, H. Kurahashi (NIID); $1 \delta^{\wedge} 1$, Gilgit, 26.vii.1987, K. Kanmiya (NIID); 3ðَ, Gilgit, 1,500 m, 26.vii.1987, S. Shinonaga \& H. Kurahashi (NIID); $5 \delta$ $3 q$, Pasu, 2,700 m, 2-3.vii.1989, H. Kurahashi (NIID); 1ठ̋, Gilgit to Pasu, Gulmit, 1,800 m, 30.vi.1989, H. Kurahashi (NIID); 1 9 , Quetta, 1,700 m, 31.vii.1990, K. Kamimura (NIID); $3 ð$ 1 , Hopel nr Gakuch, 1,900 m, 6.vii.1989, H. Kurahashi (NIID); 1ð, Gilgit to Pasu, Hussainabad, 2,000 m, 4.vii.1989, H. Kurahashi (NIID).

Distribution. Japan, Korea, Taiwan, Philippines (Leyte, Mindanao), China, India, ?Sri Lanka, Pakistan and Europe.

\section{Tribe Polleniini}

*Pollenia dasypoda Portschinsky, 1881

Specimens examined. 1, Nathiagali, 2,000 m, 10-11.vii.1989, H. Kurahashi (NIID); 2ㅇ, Karam, 2,200 m, 9.viii.1987, S. Shinonaga (NIID); 101 오, Ushu, 10 km N. Kalam, 2,800 m, 10.viii.1987, S. Shinonaga (NIID).

Distribution. *Pakistan, Arabic states, Lebanon, Syria and Europe. 
*Pollenia pediculata Macquart, 1834

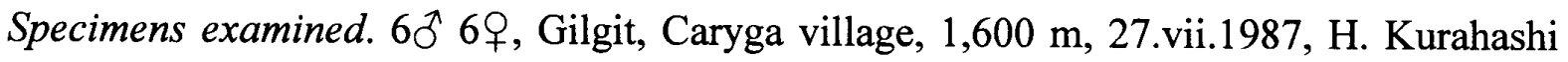
(NIID); 3ठ 1의, Gilgit, town, 1,500 m, 26.vii.1987, H. Kurahashi (NIID); 1우, Quetta, 19.ix. 1988, M. Iwasa (OUAV); 1 , Gilgit, 26.vii.1987, K. Kanmiya (NIID); 1ðَ, Ayubia-Dunga Gali, 17.vii.1987, K. Kanmiya (NIID); 19, Nathiagali, 2,000 m, 10-11.vii.1989, H. Kurahashi (NIID); $1 \delta 1$, Murtazabad, $20 \mathrm{~km}$ S of Hunza, 1,800 m, 30.vii.1987, H. Kurahashi (NIID); $1{ }^{\Uparrow} 1$, Nathiagali, $10 \mathrm{~km}$ N Murree, 2,400 m, 18.vii.1987, S. Shinonaga (NIID).

Distribution. *Pakistan.

\section{Pollenia rudis (Fabricius, 1794)}

Specimens examined. 1ठ, Gilgit, 26.vii.1987, K. Kanmiya (NIID); $1 \delta 1 \%$, nr Gilgit, 1,600 m, 8.vii.1989, H. Kurahashi (NIID); $1 \delta^{\AA} 1 q$, Ziarat, 17.ix.1988, M. Iwasa (NIID); 1q, Ushu, nr Kalam, 2,000 m, 6.viii.1988, H. Kurahashi (NIID); 3ठ 1q, Gilgit, Sharifuddinbala, 1,600 m, 28.vii.1987, H. Kurahashi (NIID); $1{ }^{\top}$, Natiagali, $10 \mathrm{~km}$ N Murree, 2,400 m, 18.vii.1987, S. Shinonaga (NIID); 2ᄋ, Nathiagali, 2,200 m, 25-30.vii.1988, H. Kurahashi (NIID); $1 \delta^{\curvearrowright}$, Naltar, $20 \mathrm{~km}$ N Gilgit, 3,000 m, 28.vii.1987, T. Inaoka (NIID); 2ðे, Murtazabad, $20 \mathrm{~km} \mathrm{~S}$ of Hunza, 1,800 m, 30.vii.1987, H. Kurahashi (NIID); 1, Swat, Miandum, 1,800 m, 7.viii.1987, K. Kanmiya (NIID); 1 , , Halter, $20 \mathrm{~km}$ W of Gilgit, 28.vii.1987, K. Kanmiya (NIID); $17 \delta_{1}$, Gilgit, town, 1,500 m, 26.vii.1987, H. Kurahashi (NIID); 21ð 1우, Gilgit, Caryga village, 1,600 m, 27.vii.1987, H. Kurahashi (NIID); 2ð, Karam, 2,200 m, 9.viii.1987, S. Shinonaga (NIID).

Distribution. Nepal, India and Pakistan. Widely distributed in the Palaearctic and Nearctic Regions.

\section{Tribe Bengaliini}

Bengalia bezzii Senior-White, 1923

Specimens examined. 20, Islamabad, 5.ix.1988, T. Hayashi (NIID).

Distribution. Japan (Ishigaki Shima I.), Taiwan, China, Vietnam, Laos, Thailand, Malaysia (Malaya), Indonesia (Java), India, Sri Lanka and Pakistan.

\section{*Bengalia escheri Bezzi, 1913}

Specimens examined. $1 \delta^{\Uparrow} 1$, Daroa, $34 \mathrm{~km} \mathrm{~s}$ of Dir, 1,800 m, 12-13.viii.1987, H. Kurahashi (NIID).

Distribution. Taiwan, China (Zhejiang, Anhui, Sichuan, Fujiang, Yunnan, Hainan), 
Malaysia (Malaya), Nepal, India and *Pakistan.

\section{*Bengalia martinleakei Senior-White, 1930}

Specimens examined. 3q, Islamabad, 5.ix.1988, T. Hayashi (NIID). Distribution. Nepal, India and *Pakistan.

*Bengalia surcoufi Senior-White, 1923

Specimens examined. 2خ, Thalkot, $25 \mathrm{~km} \mathrm{~S}$ of Bescham, 23.vii.1988, S. Shinonaga (NIID); $2 \delta 1$, , Abbottabad, 1,100 m, 31.vii.1988, H. Kurahashi (NIID); $1 \delta 3 \%$, Fort Munro, 15.ix.1988, M. Iwasa (NIID); 3ð̋, Islamabad, 5.ix.1988, T. Hayashi (NIID).

Distribution. Nepal, India (Himalayas, Assam Hills, Western Ghats) and *Pakistan.

\section{*Bengalia torosa (Wiedemann, 1819)}

Specimens examined. 1q, Fort Munro, 14.ix.1988, M. Iwasa (NIID); $1 \delta^{\lambda}$, Miandam, 1,300 m, 4.viii.1988, H. Kurahashi (NIID); 19, Mingora, 1,000 m, 3.viii.1988, H. Kurahashi (NIID); 1 9 , Abbottabad, 1,100 m, 31.vii.1988, H. Kurahashi (NIID).

Distribution. Japan (Iriomote-Jima I.), Taiwan, Philippines (Palawan, Balabac), China (Yunnan, Hainan), Laos, Thailand, Indonesia (Java), Australia (Northern Territory, ?immigrant), Nepal, India (West Bengal, Madras), Sri Lanka and *Pakistan.

\section{Bengalia unicolor Séguy, 1946}

Type material examined. Holotype $\delta$, Kurrachee, viii-ix, ? (MNHN).

Specimens examined. 1, Baluchistan, Khuzdar, 21.ix.1988, M. Iwasa (NIID).

Distribution. Pakistan.

\section{Subfamily Chrysomyinae \\ Tribe Chrysomyini}

Chrysomya albiceps (Wiedemann, 1819)

Specimens examined. 20, Chitral, 1,506 m, 8.x.1986, H. Kurahashi (NIID); 3q, Central Makran Range, Pasni, 0 m, 3-4.viii.1990, K. Kamimura (NIID); 60, 29, Kalash Valley, 1,800 m, 4.x.1986, H. Kurahashi (NIID); $1 \delta$, Chilas, 700 m, 4.viii.1987, H. Kurahashi (NIID); $1 \delta$, Quetta, 1,700 m, 31.vii.1990, K. Kamimura (NIID); 1ㅇ, Kagan Valley, Awa, 22.vii.1987, H. Kurahashi (NIID); 2ð`2 + , Hyderabad, 6.x.1986, T. Hayashi (NIID). 
Distribution. ?India and Pakistan. Widely distributed in the Afrotropical Region.

\section{Chrysomya megacephala (Fabricius, 1794)}

Specimens examined. 1, Chitral, 1,506 m, 3.x.1986, H. Kurahashi (NIID); 2q, Changa Manga Nat. Park, nr Lahore, 22.ix.1986, H. Kurahashi (NIID); 2ㅇ, D. G. Khan, 13.ix.1988, M. Iwasa (NIID); 2ठ 1q, Thatta, Haleji Lake, 10 m, 21, 23, 29.vii.1990, K. Kamimura

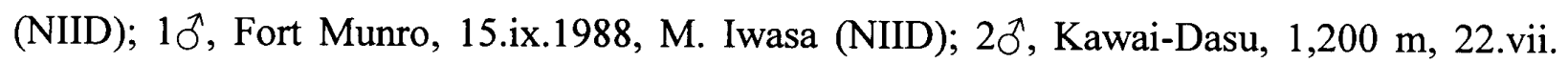
1987, H. Kurahashi (NIID); 19, Mansehra, 19.vii.1987, H. Kurahashi (NIID); $\sigma^{\curvearrowright}$, Kot Gali, 15 km W of Balakot, 1,000 m, 23.vii.1987, H. Kurahashi (NIID); 3ㅇ, Hyderabad, 6.x.1986, T. Hayashi (NIID); 1 , , Swat, Miandam, 1,800 m, 7.viii.1987, S. Shinonaga (NIID); $1 \delta^{\lambda}$, Bannu, 9.ix.1988, M. Iwasa (NIID); 1ठ, Kagan Valley, Balakot, 1,270 m, 23.vii.1987, K. Kanmiya (NIID); $1 \delta 1 q$, Ayubia-Dungagali, 17.vii.1987, K. Kanmiya (NIID); $202 \%$, Kagan Valley, Awa, 22.vii.1987, H. Kurahashi (NIID); 2ðð 19, Swat, Kalam, 1800 m, 9.vii.1987, H. Kurahashi (NIID); $1 \delta^{\lambda} 1$, , Buttgram, 1,100 m, 24.vii.1988, S. Shinonaga \& H. Kurahashi (NIID); $1 \delta 1 \%$, Kalash Valley, 1,800 m, 4.x.1986, H. Kurahashi (NIID); $\delta^{\delta}$, Abbottabad, 1,100 m, 31.vii.1988, H. Kurahashi (NIID); 1 + 15 km S of Murree, 900 m, 21.vii.1988, S. Shinonaga (NIID); 1 9 , Murree, 2,000 m, 21.vii.1988, H. Kurahashi (NIID).

Distribution. Pakistan. Throughout Oriental, Australasian and Oceanian Regions. Recently introduced to Africa, South and Central America.

\section{*Chrysomya nigripes Aubertin, 1932}

Specimens examined. 5ठ, Abbottabad, 1,100 m, 2.viii.1988, H. Kurahashi (NIID).

Distribution. Taiwan, Philippines (Luzon, Busuanga Is., Culion Is., Palawan, Samar, Mindanao, Sulu Arch.), China (Yunnan, Hainan), Vietnam, Laos, Thailand, Malaysia (Malaya), Indonesia, Nepal, India, Sri Lanka and *Pakistan.

\section{*Chrysomya phaonis Séguy, 1928}

Specimens examined. 1ठ, Kalam, 2,200 m, 9.viii.1987, S. Shinonaga (NIID); 1q, Gabral, $32 \mathrm{~km}$ N of Kalam, 7.viii.1988, S. Shinonaga (NIID); 19, Gulmit, $35 \mathrm{~km}$ N of Gilgit, 1.viiii. 1987, S. Shinonaga (NIID); 3ðَ, Chitral, 1,506 m, 3.x.1986, H. Kurahashi (NIID); 4ðे, Ayubia, 2,200 m, 22.vii.1988, H. Kurahashi (NIID); 28ð 4우, Nathiagali, 2,200 m, 25-30.vii.1988, H. Kurahashi (NIID); $5 \delta 1$, Lalazar, $10 \mathrm{~km}$ W of Naran, 2,700 m, 21.vii.1987, S. Shinonaga (NIID); $17 \delta 3$, Kagan Valley, Naran, 2,000 m, 22.vii.1987, S. Shinonaga (NIID); 2ㅇ, Swat, Kalam, 1,800 m, 9.viii.1987, H. Kurahashi (NIID); $17 \delta 2$, Kashimir, Ayubia Nat. Park, 2,200 m, 25-30.vii.1988, S. Shinonaga (NIID); 2ð, Lalazar, $16 \mathrm{~km}$ W Naran, 2,700 m, 21.vii. 1987, S. Shinonaga (NIID); 1ठ, Gilgit, Naltar, $20 \mathrm{~km} \mathrm{~N}$ of Gilgit, 3,000 m, 28.vii.1987, H. 
Kurahashi (NIID); 1ठ, Chilas, Babusar, $30 \mathrm{~km} \mathrm{~S}$ of Chilas, 3,000 m, 5.viii.1987, H. Kurahashi (NIID); 9ð, Hopel nr Gakuch, 1,900 m, 6.vii.1989, H. Kurahashi (NIID); $5 \delta 4$, Nathiagali, 2,500 m, 26-28.vi.1989, H. Kurahashi (NIID); $6{ }^{\lambda} 1$ \% , Swat, Ushu, $19 \mathrm{~km} \mathrm{~N}$ of Kalam, 2,000 m, 10.viii.1987, H. Kurahashi (NIID); 1ठ, Daroa, $34 \mathrm{~km} \mathrm{~S}$ of Dir, 1,800 m, 12-13.viii.1987, H. Kurahashi (NIID); 4ð’, Pasu, 2,700 m, 2-3.vii.1989, H. Kurahashi (NIID); 1ठึ, Gilgit to Gakuch, Gurapur, 1,600 m, 5,7.vii.989, H. Kurahashi (NIID); 2q, Kagan Valley, Awa, 22.vii.1987, H. Kurahashi (NIID); 38ð 12, Nathiagali, $10 \mathrm{~km} \mathrm{~N}$ of Murree, 2,400 m, 17-18.vii.1987, S. Shinonaga \& H. Kurahashi (NIID); 1 đ̊, 8 km E of Gilgit, 26.vii.1987, K. Kanmiya (NIID); 19ð 1 9 , Nathiagali, 2,000 m, 10-11.vii..1989, H. Kurahashi (NIID); 67 $\delta$ 12 , Kalash Valley, 1,800 m, 4.x.1986, H. Kurahashi (NIID); 5ð 1옹 Saif-ul-Malook Lake, 2,700 m, 20.vii.1987, H. Kurahashi (NIID); 2옹 Nathiagali, $10 \mathrm{~km} \mathrm{~N}$ of Murree, 2,400 m, 18.vii.1987, S. Shinonaga (NID); $2{ }^{\Uparrow} 1$ \%, Nathiagali, $10 \mathrm{~km}$ N of Murree, 2,400 m, 17-18.vii. 1987, H. Kurahashi (NIID).

Distribution. China (Sichuan, Yunnan), Tibet, Nepal, India, *Pakistan and Afganistan.

${ }^{*}$ Chrysomya pinguis (Walker, 1858)

Specimens examined. 1 , , Swat, Kalam, 1,800 m, 9.viii.1987, H. Kurahashi (NIID); 20 8우, Ayubia Nat. Park, 2,200 m, 22.vii.1988, 25-30.vii.1988, S. Shinonaga \& H. Kurahashi (NSMT); $8 ð 26 q$, Nathiagali, $10 \mathrm{~km}$ N of Murree, 2,200 m, 17-18.vii.1987, 25-30.vii.1988, S. Shinonaga \& H. Kurahashi (NSMT); 1 , Shangula Pass, 1,700 m, 9.viii.1988, H. Kurahashi (NIID); 20 2q, Nathiagali, 2,000 m, 10-11.vii.1989, H. Kurahashi (NIID); $3 \hat{\jmath}$, Nathiagali, 2,500 m, 26-28.vi.1989, H. Kurahashi (NIID).

Distribution. Japan, Korea, Taiwan, Philippines (Palawan, Tawi Tawi), China, Vietnam, Laos, Thailand, Malaysia (Malaya, Borneo), Indonesia (Java), Nepal, India (Himachal Pradesh, Assam, Madras), Sri Lanka and *Pakistan.

Chrysomya regalis Robineau-Desvoidy, 1830

Specimens examined. 2ㅇ, Fort Munro, 15.ix.1988, M. Iwasa (NIID).

Distribution. Pakistan. Wiedely distributed in the Afrotropical Region.

\section{Chrysomya rufifacies (Macquart, 1843)}

Specimens examined. 19, Ayubia Nat. Park, 2,200 m, 25-30.vii.1988, S. Shinonaga (NSMT); 2q, Chilas, 700 m, 4.viii.1987, H. Kurahashi (NIID); 2q, Kagan Valley, Awa, 22.vii.1987, H. Kurahashi (NIID); 1ठ, Daroa, 34 km S of Dir, 1,800 m, 12-13.viii.1987, H. Kurahashi (NIID); $1 \delta$, Abbottabad, 1,100 m, 2.viii.1988, H. Kurahashi (NIID); 10 , Chitral,

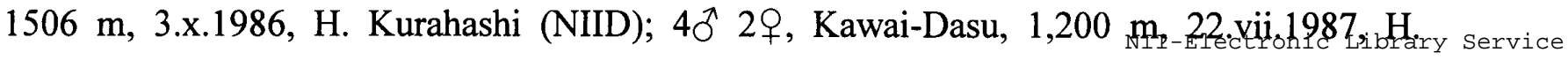


Kurahashi (NIID); 3ðં, Kalash Valley, 1,800 m, 4.x.1986, H. Kurahashi (NIID); $1 \overbrace{}^{\star}$, Swat, Ushu, $10 \mathrm{~km} \mathrm{~N}$ of Kalam, 2,000 m, 10.viii.1987, H. Kurahashi (NIID); $1 \delta, 15 \mathrm{~km} \mathrm{~S}$ of Murree, 900 m, 21.vii.1988, S. Shinonaga (NSMT).

Distribution. Pakistan. Widely distributed in the Oriental and Australasian Regions.

\section{Tribe Phromiini}

\section{*Protocalliphora azurea (Fallén, 1817)}

Specimens examined. 1q, Nathiagali, 2,200 m, 25-30.vii.1988, H. Kurahashi (NIID); $1 \delta^{\prime}$, Karam, 2,200 m, 9.viii.1987, S. Shinonaga (NIID); 19, Murree, 2,000 m, 21.vii.1988, H. Kurahashi (NIID); 20, Thandani, 2,400 m, 1.viii.1988, S. Shinonaga \& H. Kurahashi (NIID); $1 \delta$, Nathiagali, $10 \mathrm{~km} \mathrm{~N}$ of Murree, 2,400 m, 17-18.vii.1987, H. Kurahashi (NIID); 1q, Hunza, Karimabad, 2,400 m, 1.viii.1987, H. Kurahashi (NIID).

Distribution. *Pakistan. Widely distributed in the Palaearctic Region.

*Protocalliphora maruyamensis Kano et Shinonaga, 1966

The single male specimen has been examined on the hairiness of fifth sternite which somewhat differs from that of typical Japanese form, but it seems to be intraspecific variation.

Specimen examined. 1 $\delta$, Natiagali, $10 \mathrm{~km} \mathrm{~N}$ Murree, 2,400 m, 18.vii.1987, S. Shinonaga (NIID).

Distribution. Japan, Russia (Far East), China and *Pakistan.

*Protophormia terraenovae (Robineau-Desvoidy, 1830)

Specimen examined. 4ㅇ, Pasu, 2,700 m, 2-3.vii.1989, H. Kurahashi (NIID); $1 \delta$, Khunjerab Pass, 4,700 m, 2.viii.1987, H. Kurahashi (NIID).

Distribution. *Pakistan. Widely distributed in the Palaearctic and Nearctic Regions.

\section{Subfamily Rhiniinae \\ Tribe Rhiniini}

*Stomorhina cribrata (Bigot, 1874)

Specimens examined. 1ठ, Quetta, 19.ix.1988, M. Iwasa (NIID).

Distribution. *Pakistan, Arabic states, Israel and Iran. Widely distributed in the Afrotropical Region. 


\section{Stomorhina discolor (Fabricius, 1794)}

Specimens examined. 1ㅇ, Ziarat, 17.ix.1988, M. Iwasa (OUAV); $2 \delta 2 ᄋ$, Nathiagali, 10 km N of Murree, 2,400 m, 17-18.vii. 1987, H. Kurahashi (NIID); 29, Chilas, 4.viii.1987, K. Kanmiya (NIID); 1 ㅇ, Miandum, 1,800 m, 7.viii.1987, S. Shinonaga (NIID); $1 \delta 3$ 연 Ayubia Nat. Park, 2,200 m, 25-30.vii.1988, S. Shinonaga (NIID); 1ठ, Thalkot, 25 km S. Besham, 23. vii.1988, S. Shinonaga (NIID); 1 $\widehat{ }$, Manshera, 1,000 m, 2.viii.1988, S. Shinonaga (NIID); $6{ }^{\star}$ 1오. Swat, Kalam, 1,800 m, 9.viii.1987, H. Kurahashi (NIID); 4 $\delta$ 3오. Nathiagali, 2,000 m, 10-11.vii.1989, H. Kurahashi (NIID); 3ठ, Nathiagali, 2,200 m, 25-30.vii.1988, H. Kurahashi (NIID); 1ठ, Ushu, nr Kalam, 2,000 m, 6.viii.1988, H. Kurahashi (NIID); 1ð, Kalam, 1,800 m, 5.viii.1988, H. Kurahashi (NIID); $10^{\star}$, nr Abbottabad, 1,300 m, 28.vi.1989, H. Kurahashi (NIID); 2ठ, Swat, Marghazar, 1,400 m, 8.viii.1987, H. Kurahashi (NIID); 21 $ત 8 q$, Nathiagali, 2,500 m, 26-28.vi.1989, H. Kurahashi (NIID).

Distribution. Taiwan, Philippines, China, Vietnam, Laos, Thailand, Malaysia, Indonesia (Buru I.), Australia (Northern Territory), New Caledonia, Fiji, India, Sri Lanka and Pakistan. Widely distributed in the Indo-Australian region.

*Stomorhina procula (Walker, 1849)

Specimens examined. $1 \delta$, Tret, $22 \mathrm{~km} \mathrm{~S}$ of Murree, $500 \mathrm{~m}$, 26.vi.1989, H. Kurahashi (NIID); $21 \delta^{\wedge} 7$, Nathiagali, 2,500 m, 26-28.vi.1989, H. Kurahashi (NIID); $5 \delta$ 8웅, Nathiagali, 2,000 m, 10-11.vii.1989, H. Kurahashi (NIID); 2^ 11 , Nathiagali, 2,200 m, 25-30.vii.1988, H. Kurahashi (NIID); $3 ð 2$, Nathiagali, 2,400 m, 17-18.vii.1987, H. Kurahashi \& S. Shinonaga (NIID); 1ð, Ayubia, 2,200 m, 22.vii.1988, H. Kurahashi (NIID).

Distribution. China, Vietnam, Laos, Malaysia (Malaya, Sarawak), Myanmar, Nepal, India (Madras, Coonoor) and *Pakistan.

\section{Tribe Cosminini}

\section{${ }^{*}$ Cosmina prasina (Brauer et Bergenstamm, 1889)}

Specimens examined. 1q, Karachi, 23.ix.1988, M. Iwasa (NIID); 3ठ, Tandani, 26 km N. Abbottabad, 2,400 m, 1.viii.1988, S. Shinonaga (NIID).

Distribution. *Pakistan, Egypt, Italy and North Africa. Widely distributed in the Oriental and Afrotropical Regions. 
*Isomyia aurifacies James, 1970

Specimens examined. $3 \delta^{\lambda} 1 q$, Buttgram, 1,100 m, 24.vii.1988, H. Kurahashi \& S. Shinonaga (NIID); 1q, Manshera, 1,000 m, 2.viii.1988, S. Shinonaga (NIID); 2q, Abbottabad, 1,100 m, 2.viii.1988, H. Kurahashi (NIID).

Distribution. Vietnam, India (Assam) and *Pakistan.

*Isomyia fulvicornis (Bigot, 1887)

Specimens examined. 1ð, Daroa, $34 \mathrm{~km} \mathrm{~S}$ of Dir, 1,800 m, 12-13.viii.1987, H. Kurahashi (NIID); 1, Swat, Marghazar, 1,400 m, 8.viii.1987, H. Kurahashi (NIID); 2ㅇ, Mingora, 1,000 m, 8-10.viii.1988, H. Kurahashi (NIID).

Distribution. Vietnam, Malaysia (Malaya, Borneo), Indonesia (Java), India, Sri Lanka and *Pakistan..

*Isomyia pseudoviridana (Peris, 1952)

Specimens examined. $6 \bigcirc^{\AA} 2$, Natiagali, 2,400 m, 18.vii.1987, S. Shinonaga (NIID); 1 , Balakot, 1,000 m, 23.viii.1987, S. Shinonaga (NIID); 1ठ, Battagram, 1,400 m, 24.vii.1988, S. Shinonaga (NIID); 1ð, Tret, $22 \mathrm{~km} \mathrm{~S}$ of Murree, $500 \mathrm{~m}, 26 . v i .1989$, H. Kurahashi (NIID); 1 , Mohashrif, $50 \mathrm{~km} \mathrm{~S}$ of Dir, 12.viii.1987, H. Kurahashi (NIID); 2q, Nathiagali, 2,500 m, 26-28.vi.1989, H. Kurahashi (NIID); 7ठ 17q, Nathiagali, $10 \mathrm{~km} \mathrm{~N}$ of Murree, 2,400 m, 17-18.vii.1987, H. Kurahashi (NIID); 90 13, Nathiagali, 2,500 m, 26-28.vi.1989, H. Kurahashi (NIID).

Distribution. China (Anhui, Zhejian, Sichuan, Fuijian, Guangdon, Hainan), Myanmar, Nepal, India (Assam), Sri Lanka, and * Pakistan.

Rhyncomya townsendi James, 1977

Specimens examined. 19, Ziarat, 16.ix.1988, M. Iwasa (NIID); 1q, Central Makeran Range, Jiwani, 8-9.viii.1990, K. Kamimura (NIID).

Distribution. Pakistan.

\section{DESCRIPTION}

Onesia kiyoshii sp. nov.

(Fig. 1) 
distance slightly more than width of anterior ocellus; frons index 0.02-0.06 (mode 0.04, $\mathrm{n}=$ 18); frontal stripe black, not obliterated at narrowest point; parafrontalia and parafacialia black, grey-dusted, setulose, setulose hairs black, fine, rather long; face black, dark-grey dusted, with trace of median carina between bases of antennae; facialia largely blackish except for outer margin brownish, with black setulae on lower one-third; mediana dark red, with some black setulae on lower part close to gena; vibrissaria narrow, dark red, with a few fine setulae; vibrissa well developed; epistome black, dark-grey dusted, not projecting forward; gena black, dark-grey dusted, clothed with black hairs; postgena black, dark-grey dusted, largly covered with black hairs except for a few yellowish ones; occiput concolorous with gena, with yellowish hairs on lower and central parts; 2nd antennal segment black, subshining, with some black setulae and one rather long bristly hair; 3rd antennal segment blackish, dark-grey pubescent, $3 \times$ as long as 2nd; arista dark brown, blackish basally, long-plumose; palpus yellowish brown.

Thorax: black, dark-grey dusted, two median narrow and two lateral broad longitudinal stripes marked on prescutum, lateral one not interrupted just behind internal $p h$; humerus and postalar callus concolorous with thoracic dorsum; scutellum concolorous with thoracic dorsum; prosternum and propleuron with black hairs; supraspiracular convexity pubescent; hypopleural hairs black; other pleural hairs black; mesothoracic spiracle pale brown, but largely blackish along margin; metathoracic one blackish; postalar declivity with rather long black hairs in central circle; tympanic and anterior parasquamal tufts both consist of blackish hairs. Chaetotaxy: ac 1-2+3; dc 2+3; ia $0+2$; h 3; ph 1; prs 1 ; sa 3; pa 2; st 2+1; sc 3+1; n 2; pp 1-2; pst 1 .

Wings: hyaline; veins brown; epaulet blackish; basicosta blackish; subcostal sclerite largely dark brown except for base yellowish; node of 2 nd and 3 rd longitudinal veins with a few black setulae above and below; 4th longitudinal vein bending forward at right angle; section of 4th vein from bend to wing edge inflexed and then nearly straight; R5 open; squamae totally brownish, alar one yellowish on anterior part and with tuft of blackish-brown hairs at inner lower margin; thoracic squama with patch of black hairs on basal half. Halteres brown, with yellowish knob.

Legs: black; fore tibia with 3-4 short $a d$ and 1 strong $p$; mid tibia with $1 \mathrm{ad}, 2 \mathrm{pd}$ and $1 v$; hind tibia 2-3, usually 2 strong $a d, 2 p d$ and $2 a v$.

Abdomen: metallic blue to green, more or less silver-grey dusted, with trace of median dark longitudinal stripe on tergites 3 and 4; abdominal hairs black; tergite 1+2 with some lateral marginal bristles and bristly hairs on lateral side; tergite 3 with row of marginal bristles; tergites 4-5 with row of long erect marginal bristles, some fine erect bristly hairs on disc; sternites black, clothed with black hairs and bristles; no remarable hairiness on sternites and lateral sides of tergites. Hypopygium normal in size, male genitalia as shown in Fig. 1.

우.-Head: dichoptic; eyes separated at vertex by about $4 \mathrm{X}$ width of ocellar triangle; frons index 0.32-0.35 (mode 0.33, $\mathrm{n}=6$ ); frontal stripe broad, almost parallel-sided,-dark red, more $_{\text {ry }}$ service 
Med. Entomol. Zool.

darkened toward vertex, width slightly less than $4 \mathrm{X}$ that of one of parafrontalia just in front of anterior ocellus; parafrontalia black, dark-grey dusted, with black setulae, provided with ca 7 pairs of ori; ors 2+1; oc developed; acoc fine; pooc divergent; $o v$ and $i v$ well developed; $p o c$
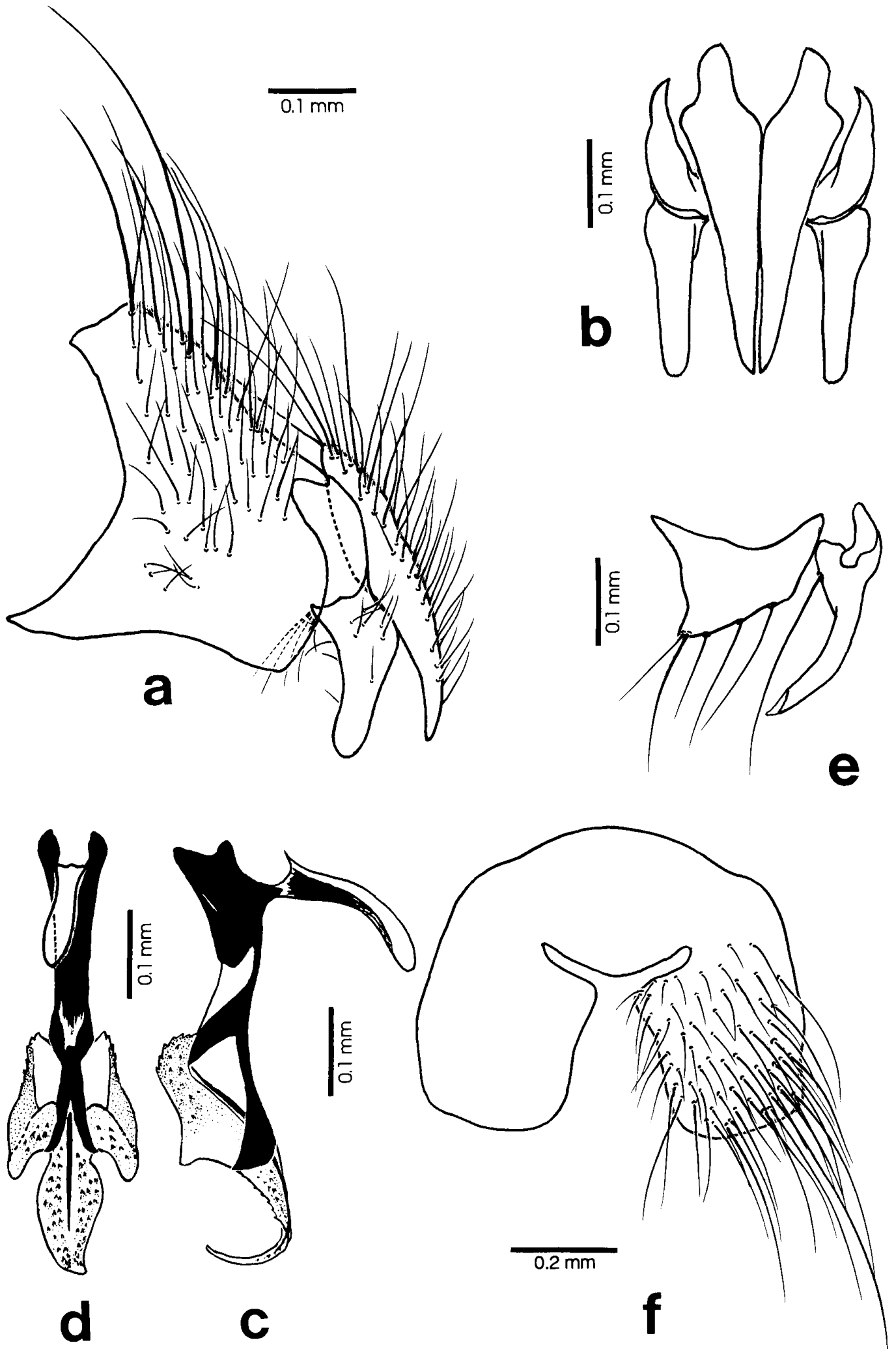

Fig. 1. Onesia kiyoshii sp. nov., male genitalia.- a: Epandrium, cercus and paralobus, lateral view; b, cerci and paralobi, caudal view; c, aedeagus, lateral view; d, aedeagus, posterior view; e, anterior and posterior parameres, lateral view; f, sternite 5 , ventral view. 
parallel or divergent; occ absent. Thorax: bristles more stronger than those of $\bar{\sigma}$. Wings: similar to those of $\delta$. Legs: fore tibia with 1-2 short ad; mid tibia with 1-2 ad; hind tibia with 1-2 av. Abdomen: bristles less developed than those of $\delta$, decumbent. Ovipositor short. Otherwise same as for $\bar{\delta}$.

Length: 4.2-6.5 $\mathrm{mm}$.

Type material. Holotype $\delta$, " Holotype [red card]// PAKISTAN:/ NWF Prov., $2200 \mathrm{~m} /$ Nathiagali/ 25-30.VII.1988/ Col. H. Kurahashi//Onesia/ kiyoshii/ sp. nov./ Det. H. Kurahashi [red perimeter]." Paratypes. $12 \AA 4 \%$, same data as holotype, except "Paratype [yellow card] and //Onesia/ kiyoshii/ sp. nov./ Det. H. Kurahashi [yellow perimeter]"; $3 \delta 1$, [yellow card]// PAKISTAN:/ NWF Prov., 2400m/ Thandani/ 1.VIII.1988/ Col. H. Kurahashi//Onesia/ kiyoshii/ sp. nov./Det. H. Kurahashi [yellow perimeter]"; $10 \AA$, " Paratype [yellow card]// PAKISTAN: NWF Prov./ Ayubia, $2200 \mathrm{~m} / 22$.VII..1988/ Col. H. Kurahashi//Onesia/ kiyoshii/ sp. nov./ Det. H. Kurahashi [yellow perimeter]"; $1 \delta^{\lambda} 10$, " Paratype [yellow card]// PAKISTAN/ Ayubia Nat./ Park, 2200 m/ Kashmir/ 25-30 July 1988/ S. Shinonaga// Onesia/ kiyoshii/ sp. nov./ Det. H. Kurahashi [yellow perimeter]."

Etymology. The specific name is in honor of Dr. Kiyoshi Kamimura (Chief of the Survey), Toyama Medical and Pharmaceutical University, Toyama.

Type depository. Holotype $\widehat{\sigma}$ and paratypes $(14 \hat{\sigma} 3 q)$ are deposited in NIID. Paratypes $(1 \delta 1 \%)$ in NSMT, BPBM and BMNH, respectively.

Remarks. This new species is very similar to Onesia atripalpis (Malloch, 1935) in the general appearance, but cen be easily distinguished by the presence of presutural $i a$ and the genitalic evidence.

Bionomics. The adult flies were found on flowers in mountainous areas.

Distribution. Pakistan.

\section{ACKNOWLEDGEMENTS}

We are indebted to Dr. K. Kamimura (Chief of the Survey), Toyama Medical and Pharmaceutical University, Toyama, allowing me to carry out this study. We thank the members of the survey, Drs. S. Shinonaga, K. Saito, T. Inaoka, K. Kanmiya, M. Iwasa and T. Hayashi, for offering us the relevant material from their collections. Thanks are also Dr. K. Rognes for his assistance in the identification of Pollenia spp.

This study was supported by the Grant-in-Aid, for Overseas Scientific Survey from the Ministry of Education, Science and Culture, Japan, entitled "Zoogeographical Studies on the Medically Imortant Diptera in South-West Asia" (Nos. 61042004, 62041036 and 63041058). 


\section{REFERENCES}

Fan, Z. 1965. Key to the Common Synanthropic Flies in China. xv+330 pp Academia Sinica, Peking. (in Chinese).

James, M. T. 1977. Family Calliphoridae. In: Delfinado, M. D. and D. E. Hardy (ed.). A catalog of the Diptera of the Oriental Region, Volume III. Suborder Cyclorrapha (excluding Division Aschiza). 526-556 pp. The University Press of Hawaii, Honolulu.

Senior-White, R., Aubertin, D. and Smart, J. 1940. The fauna of British India, including remainder of the Oriental region. Diptera VI. Family Calliphoridae. xiii +288 pp. Taylor and Francis Ltd., London. 